\title{
Production of capsular polysaccharide does not influence Staphylococcus aureus vancomycin susceptibility
}

\author{
Andrea Jansen ${ }^{1}$, Christiane Szekat ${ }^{1}$, Wiebke Schröder ${ }^{2}$, Christiane Wolz ${ }^{2}$, Christiane Goerke², Jean C Lee ${ }^{3}$, \\ Michael Türck' and Gabriele Bierbaum ${ }^{1 *}$
}

\begin{abstract}
Background: Diverse mechanisms (increased cell wall thickness, low cross linking, decreased autolysis, etc.) have been reported for Staphylococcus aureus strains with intermediate vancomycin susceptibility (VISA). This study was conducted to identify common mechanisms responsible for decreased vancomycin susceptibility in a VISA strain pair.

Results: Transcriptional profiling of the clinical heterogeneous VISA isolate SA137/93A and its spontaneous homogeneous mutant strain SA137/93G pointed to an increased capsule production in the strain pair compared to a susceptible control. Furthermore, transcript quantification of the gene cap5E, which is essential for capsule biosynthesis, revealed elevated levels in the VISA strains SA137/93A, SA137/93G and Mu50 in comparison with susceptible strains Reynolds, Newman and SA1450/94. The increased expression was observed in bacteria from exponential as well as stationary growth phase. However, suppression of type 5 capsule formation by expression of antisense RNA did not increase vancomycin susceptibility in the VISA strain SA137/93G. Likewise, construction of inducible mutants of S. aureus Newman or repair of capsule biosynthesis of S. aureus HG001 and S. aureus 1450/94 did not influence resistance to vancomycin. Furthermore, purified type 5 polysaccharide did not protect indicator strains from the action of vancomycin.

Conclusions: The VISA strain tested in this study displayed an increased production of type 5 capsular polysaccharide. However, the production of capsule material did not protect strain SA137/93G and three vancomycin sensitive strains in the presence of vancomycin and thus is not part of the resistance mechanism; however it may represent a by-product of VISA life style that is often characterized by a high sigma factor B activity.
\end{abstract}

\section{Background}

The phenotype "intermediate vancomycin resistance" in Staphylococcus aureus (CLSI: MIC $=4-8 \mathrm{mg} / \mathrm{L}$ in Mueller Hinton broth $(\mathrm{MH})$ ) has been assigned to changes that lead to alterations in cell wall synthesis and morphology. Most vancomycin intermediately resistant $S$. aureus (VISA) strains are characterized by increased cell wall thickness as a consequence of activated cell wall biosynthesis and decreased autolysis [1-7]. The mechanism of resistance was shown to be based on an enhanced amount of free D-Ala-D-Ala termini in the cell wall, which act as

\footnotetext{
* Correspondence: Bierbaum@mibi03.meb.uni-bonn.de

'Institut für Medizinische Mikrobiologie, Immunologie und Parasitologie, Universitätsklinikum Bonn, Sigmund-Freud-Str. 25, D-53105, Bonn, Germany Full list of author information is available at the end of the article
}

false target sites that keep the vancomycin molecules from reaching lipid II $[2,8]$. Many studies have attempted to elucidate the genetic basis of this resistance type, mainly by comparative transcriptional profiling and full genome sequencing (for a review see [9]). Transcriptional profiling revealed a variety of genes that were differentially expressed in VISA strains or resistant laboratory mutants (MIC: $\geq 32 \mathrm{mg} / \mathrm{L}$ ) in comparison with vancomycin susceptible $S$. aureus (VSSA). From these results it was postulated that an activated sugar and lipid metabolism and increased energy are required to generate thicker cell walls in VISA strains [10-12]. Furthermore, mutations in two component regulatory systems $(y y c F G$, which was recently renamed walKR, yvqF/vraSR and graRS) are assumed to play a central role in adaptation to the antibiotic stress

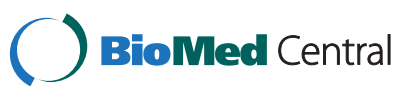


[9,13-19], as well as mutations in $r p o B$ [20-22], pknB [23], prsA [24] and clpP [25].

The clinical methicillin resistant VISA isolate SA137/93A was isolated from a tracheal secretion and displays heterogeneous intermediate vancomycin resistance (hVISA strain, MIC: $2 \mathrm{mg} / \mathrm{L}$ in $\mathrm{MH}, 8 \mathrm{mg} / \mathrm{L}$ in brain heart infusion (BHI)). Subculturing in the presence of $6 \mathrm{mg} / \mathrm{L}$ vancomycin generated a mutant with homogeneous intermediate vancomycin resistance, which showed an MIC value of $16 \mathrm{mg} / \mathrm{L}$ in BHI (4 mg/L in $\mathrm{MH}$ ) and was designated SA137/93G [4]. Pulsed-field gel electrophoresis (PFGE) profiles, phage typing and MLST sequencing of the strains showed that they were members of the Iberian clone (ST247) which was prevalent in Germany in the early 1990's under the designation "Northern German epidemic strain". Both strains possess a thickened cell wall [4]. The decreased vancomycin susceptibility of strain SA137/93A is most probably based on an increased amount of free D-Ala-D-Ala termini in the cell wall, which is due to decreased crosslinking. Surprisingly, the cell wall cross linking of strain SA137/93G was within the standard range [4]. As a first step in analysis of the genetic background of the decreased vancomycin susceptibility of both strains, the insertion patterns of the highly mobile insertion element IS256 were compared and found to be different. Strain SA137/93G is characterized by an insertion of IS256 into the gene tcaA [26,27] and reconstitution of tcaA led to a decrease in vancomycin resistance. In contrast, strain SA137/93A displays an IS256 insertion in the promoter region of the essential two-component system $y y c F G$ (walRK) which leads to an increased expression of this system [27]. However, although both insertions were shown to correlate with a decrease in susceptibility to vancomycin, the difference in the vancomycin resistance level of the strain pair could be mainly attributed to the disruption of $t c a A$ in SA137/93G [27]. Furthermore, SA137/93G carries a deletion which starts at the IS431 element at the left junction of the SCCmec and covers a chromosomal fragment that comprises SA0027 to SA0132 [4]. Similar deletions starting at the very same bp have been described for MRSA strains after storage in the laboratory [28]. The absence of mecA also contributed to the higher vancomycin resistance of strain SA137/93G [4].

This study was conducted to identify common mechanisms responsible for decreased vancomycin susceptibility in the hVISA isolate SA137/93A and its homogeneous resistant derivative SA137/93G. To this end, we compared the transcriptomes of both strains with that of the closely related vancomycin susceptible MRSA strain SA1450/94 (MIC: $2 \mathrm{mg} / \mathrm{L}$ in BHI). We found that the genes encoding capsule biosynthesis were the only genes with higher expression in both VISA strains and therefore tested whether the staphylococcal type 5 capsular polysaccharide (CP5) might be involved in vancomycin resistance of this strain.

\section{Methods}

Bacterial strains, plasmids, growth conditions and antimicrobial susceptibility testing

Bacterial strains and plasmids are listed in Table 1. S. aureus strains were cultured in BHI medium, containing $2 \mathrm{~g} / \mathrm{L}$ glucose (Becton Dickinson GmbH, Heidelberg, Germany) at $37^{\circ} \mathrm{C}$ with aeration, unless indicated otherwise. For every experiment an overnight culture was diluted 100-fold in fresh BHI broth and further incubated to an optical density at $600 \mathrm{~nm}\left(\mathrm{OD}_{600}\right)$ of 0.8-1.0 to ensure exponential growth conditions. Determination of vancomycin MICs was performed following the microdilution method according to CLSI guidelines except using BHI medium unless indicated otherwise. For E-testing cultures were diluted to an optical density of 2 McFarland and plated on BHI agar. Population analysis was carried out as described previously [29]. Some antisense experiments were performed on TSA without glucose (TSA-G). Antisense plasmids were selected with $34 \mu \mathrm{g} / \mathrm{ml}$ chloramphenicol $(\mathrm{CM})$ as described for the system [30]; otherwise $20 \mu \mathrm{g} / \mathrm{ml}$ of chloramphenicol was employed.

\section{Comparative transcriptomics}

For transcriptional profiling, the strains compared were grown to an $\mathrm{OD}_{600}$ of 0.8-1.0. Preparation of total RNA, cDNA synthesis and fluorescence labelling as well as microarray experiments using the sciTRACER S. aureus N315 full genome chip (Scienion AG, Berlin, Germany) were performed as described previously [27]. The respective experiments were replicated at least four times including a dye swap. The microarray data were deposited in the gene expression omnibus (GEO) database at NCBI under accession number GSE10529.

\section{Comparative genomics}

Genomic DNA of the strains SA137/93A, SA137/93G and SA1450/94 was prepared employing genomic tip 20 columns (Qiagen, Hilden, Germany) according to the manufacturer's instructions. Cell lysis was supported by incubating the cell suspension for $1 \mathrm{~h}$ at $37^{\circ} \mathrm{C}$ in the presence of $50 \mathrm{mg} / \mathrm{L}$ lysostaphin. Genomic DNA (3 $\mu \mathrm{g})$ was labelled using the Bioprime DNA labelling system (Invitrogen, Karlsruhe, Germany) following the instruction manual. The labelling reaction was performed in the presence of $0.1 \mathrm{mM}$ cyanine-3'- or cyanine-5'-labelled dCTP (Perkin Elmer Life Science, Mechelen, Belgium) in addition to $0.2 \mathrm{mM}$ dCTP, $0.5 \mathrm{mM}$ dATP, $0.5 \mathrm{mM}$ dGTP and $0.5 \mathrm{mM}$ TTP. The labelled DNA was purified using the MinElute purification kit (Qiagen) and subsequently compared by competitive hybridisation employing the sciTRACER S. aureus N315 full genome chip as described previously [27]. The experiment was conducted in duplicate including a dye swap. 
Table 1 Strains and plasmids

\begin{tabular}{|c|c|c|}
\hline Strain/plasmid & Relevant genotype or phenotype $^{a}$ & Source/reference \\
\hline \multicolumn{3}{|l|}{ S. aureus strains } \\
\hline SA137/93A & $\begin{array}{l}\text { Clinical hVISA isolate; MET', Northern German epidemic MRSA, MLST sequence } \\
\text { type ST247 }\end{array}$ & [4] \\
\hline SA137/93G & Mutant VISA of SA137/93A; $\triangle$ SCCmec $\left(\right.$ MET $\left.^{\text {S }}\right)$, ST247 & {$[4,27]$} \\
\hline $\begin{array}{l}\text { SA137/93G } \\
\text { pCapDvorne }\end{array}$ & SA137/93G carrying pCapDvorne $\left(\mathrm{CHL}^{\prime}\right)$ & This work \\
\hline Mu50 & Clinical VISA isolate & [29] \\
\hline SA1450/94 & $\begin{array}{l}\text { Northern German epidemic MRSA, Iberian clone, ST247, CP5 positive, VAN } \\
\text { control }\end{array}$ & $\begin{array}{l}\text { German reference centre for staphylococci, } \\
\text { Wernigerode }\end{array}$ \\
\hline SA1450/94 pCapAre & SA1450/94, repair of cap5A & This work \\
\hline SA1450/94 pCU1 & SA1450/94, control (empty vector) & This work \\
\hline Newman & CP5 positive, VAN ${ }^{5}$ & NCTC8178 \\
\hline Newman-132 & pMUTIN integrated into capsule promoter & This work \\
\hline NCTC8325 (RN1) & laboratory strain & National collection of type cultures \\
\hline HG001 & NCTC8325, repaired in rsbU & [31] \\
\hline HG001 pCap5E & HG001 capsule production repaired & This work \\
\hline SG511-Berlin & sensitive antibiotic screening strain & {$[32]$} \\
\hline RN4220 & Restriction negative derivative of NCTC8325, cloning host & [33] \\
\hline \multicolumn{3}{|l|}{ Plasmids } \\
\hline pUC19gyrB & $\begin{array}{l}\text { pUC19, carrying a } 560 \text { bp internal fragment of gyrB; external plasmid standard } \\
\text { for real time PCR }\end{array}$ & This work \\
\hline pCU1cap5E & pCU1 (Augustin), carrying cap5E; external plasmid standard for real time PCR & This work \\
\hline pCapDvorne & pEPSA5 (Forsythe) harbouring a cap5D antisense fragment & This work \\
\hline pCapAre & pCU1 harbouring cap $5 A$ & [34] \\
\hline pCap5E & pCU1 harbouring cap5E & [34] \\
\hline pCG132 & pMUTIN4 (Vagner 1998) habouring cap5A & This work \\
\hline
\end{tabular}

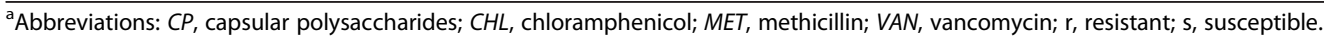

\section{Immunofluorescence labelling of CP5}

The incubation time and media employed for capsule production are indicated in the figure legends. CP5 production was detected by an indirect immunofluorescence technique [35]. In brief, bacteria were fixed to microscope slides with heat and incubated for one hour with human serum to saturate protein A. The human serum had been pretreated with protein A deficient strain Newman (diluted 1:10 in PBS with 0.05\% Tween $20)$ to remove existing $S$. aureus antibodies from the serum. Slides were washed and incubated for $1 \mathrm{~h}$ at ambient temperature with rabbit antiserum specific for CP5 and diluted 1:200 in PBS with 0.05\% Tween 20. The slides were again washed three times before incubation with $\mathrm{CY} 3$-conjugated goat $\mathrm{F}(\mathrm{ab})_{2}$ fragments raised to rabbit IgG (Dianova, Hamburg, Germany) diluted 1:500 in PBS with $0.05 \%$ Tween 20. In a subsequent step, the bacteria were stained with 4,6-diamidino-2phenylindol (DAPI, $2 \mathrm{mg} / \mathrm{L}$; Sigma-Aldrich, Munich, Germany) for $5 \mathrm{~min}$ at room temperature.
Transcript quantification by real time PCR

Cells of the VISA strains SA137/93A and SA137/93G and the susceptible controls SA1450/94 and Newman (the CP5 type strain) were harvested from a culture at $\mathrm{OD}_{600} 0.3,0.5$, 1, 2 and 4-5. RNA preparation and cDNA synthesis were done as previously described [27]. Experiments were conducted at least in duplicate for each strain. Transcript amounts of cap5E were determined in LightCycler (Roche Diagnostics, Mannheim, Germany) experiments by quantification relative to the housekeeping gene $\operatorname{gyr} B$ employing external plasmid standards (Table 1) as described previously [27]. PCR experiments were conducted using the LightCycler FastStart DNA Master SYBR Green I Kit (Roche Diagnostics, Mannheim, Germany) according to the manufacturer's instructions and the gene specific primer pairs gyrB-1-RT and gyrB-2-RT [27] and cap5E-1-RT (CCAGTTGAGGCAGTGAAGACA; NCBI: NC_002745 bp 171655-676) and cap5E-2-RT (CTGATCCTCTTGAA GCCATCAC; NCBI: NC_002745 bp 171878-899), respectively. The following temperature profile was utilized for 
amplification: Initial denaturation at $95^{\circ} \mathrm{C}$ for 10 minutes $\left(20^{\circ} \mathrm{C} / \mathrm{s}\right) .45$ cycles of denaturation $\left(95^{\circ} \mathrm{C} ; 1 \mathrm{~s} ; 20^{\circ} \mathrm{C} / \mathrm{s}\right)$, annealing $\left(55^{\circ} \mathrm{C} ; 15 \mathrm{~s} ; 20^{\circ} \mathrm{C} / \mathrm{s}\right)$, elongation $\left(72^{\circ} \mathrm{C} ; 15 \mathrm{~s}\right.$; $20^{\circ} \mathrm{C} / \mathrm{s}$; single mode). Specificity of the PCR reaction was verified by melting curve analysis $\left(45^{\circ} \mathrm{C}\left(10 \mathrm{~s} ; 20^{\circ} \mathrm{C} / \mathrm{s}\right)\right.$ to $95^{\circ} \mathrm{C}\left(0.2^{\circ} \mathrm{C} / \mathrm{s}\right)$, continuous mode) and ethidium bromide staining on agarose gels. Calculation was done by the second-derivative maximum method. The quantification assays were conducted employing RNA prepared from two independent cultures of each strain.

\section{Antisense experiments}

A 166 bp fragment located in the N-terminus of cap5D was amplified using the primers capD-vorne-166_antifor (AAATCTAGAATCTGTGAAATTGCGGCTTT) and capD-vorne-166_anti-rev (AAAGAATTCTGCTGAAATA TGATGCGATATG) with Phusion DNA polymerase (New England Biolabs, Frankfurt, Germany) and ligated to the vector pEPSA5 [30] using the XbaI and EcoRI restriction sites. The ligation assay was transformed into $E$. coli JM83 by electroporation, the recombinant plasmid was shuttled into S. aureus RN4220 by electroporation [36] and subsequently transduced into S. aureus SA137/93G by phage transduction using bacteriophage $80 \alpha$ [37]. For expression of antisense RNA, the cultures were grown in LB (lysogeny broth)/CM34 or other media as indicated [30] and were divided for addition of $50 \mathrm{mM}$ xylose to one of the cultures. Sequencing confirmed that pEPSA5 does not contain the cre sequence, which would inhibit transcription in the presence of glucose.

\section{Complementation of cap5E}

The defect in Cap5E in strains of the NCTC 8325 lineage (the M134R exchange that leads to inactivation of the protein) was complemented using cap5E on pCU1 as described in [34]. The DNA fragment harbouring cap5E (bp 3394-5448 in NCBI acc. nr. U81973, [34]) was amplified by PCR employing the primers cap5Eforward (GCTTCTAGA CTAGTTTTGCAGGCAGG) and cap5Ereverse (GTCGA GCTCGTTAAATCTGCTTTCAA) from $S$. aureus Newman DNA, ligated into pCU1 and after subcloning in E. coli and S. aureus RN4220 the recombinant plasmid was introduced into S. aureus HG001 [31].

\section{Generation of a conditional capsule mutant}

In gram-positive bacteria, pMUTIN4 is an integrative vector that places the downstream genes under control of a Pspac promoter [38]. The cap5A gene was amplified using the primers EcoRIcap431-for (GGGGGAATTCTAAGGGA GGTAAATAATGG) and BamHcap1431-rev (ACACGGAT CCATTAAGCTTGATAGTCCA), ligated with the restricted (EcoRI, BamHI) pMUTIN4 plasmid and transformed into E. coli. The resulting plasmid (pCG132) was verified by sequencing and electroporated into $S$. aureus strain
RN4220. Since pMUTIN4 does not have a gram-positive origin of replication, all clones had gone through a single crossover event, which inserted the vector into the genome and placed the cap $5 \mathrm{~A}$ gene under the control of the IPTG-inducible Pspac promoter. The integrated plasmid was then transduced into strain Newman using $\Phi 11$ lysates. Mutants were verified by PCR using the oligonucleotides P5spac (TACATCCAGAACAACCTCTG) and capArev (GACTTTAACTGCTGTACCGTCTGCT) and PFGE.

\section{Extraction of capsular polysaccharides (CP)}

For extraction of crude capsule extract, staphylococci were plated onto Columbia blood agar plates that had been supplemented with $50 \mathrm{mM} \mathrm{NaCl}$. After $24 \mathrm{~h}$ of incubation at $37^{\circ} \mathrm{C}$, the bacteria were harvested by suspension in PBS buffer. The CP was detached from the cells by autoclaving at $120^{\circ} \mathrm{C}$ for $1 \mathrm{~h}$ and the cell debris was removed by centrifugation. The supernatant was passed through a cellulose acetate filter (pore size $0.45 \mu \mathrm{m}$ ). Cell wall teichoic acid was removed by treatment with $50 \mathrm{mM} \mathrm{NaIO}$ for $72 \mathrm{~h}$ at room temperature in the dark [39]. The crude extract was then washed with PBS buffer by ultrafiltration on a YM10 membrane (Millipore, Schwalbach, Germany) or employing Vivaspin 6 columns (exclusion volume of $3 \mathrm{kDa}$ ) (Sartorius, Göttingen Germany). These extracts were then added to MIC determinations in $\mathrm{MH}$ medium using $S$. aureus NCTC 8325 and S. aureus SG511 as indicator strains. In order to test for contaminating nucleic acids, the extracts were digested with DNase and RNAse [40] and tested again. Crude capsule extract from S. aureus NCTC 8325 which cannot produce a capsule because of the point mutation in Cap5E and PBS buffer served as negative controls in these experiments. Purified CP5 was obtained as described in [41].

\section{Sequencing of the promoter region of the CP5 biosynthesis gene cluster}

A 735 bp DNA segment comprising the promoter region of the CP5 biosynthesis gene cluster was amplified using a standard PCR protocol and the primer pair (AGCTCG CATTTGAAGATCAATGT) and (CCTCTTGTGCCATA AACTGAGG) (bp 166966-166988 and bp 167586-167607, NCBI: NC_002745). The product was purified (QIAquick Gel Extraction Kit, Qiagen, Hilden, Germany) and sequenced (Sequiserve, Vaterstetten, Germany).

Detection of the cap5 gene cluster in the VISA strains was performed using primers cap5-9864 (GTACGAAGCG TTTTGATAGTT) and cap5-9332 (GAAAGTGAACGAT TAGTAGAA) that flank the type-specific sequences of cap5I and cap5J in S. aureus [42].

The insertion of IS256 in cap5A in S. aureus SA1450/94 was complemented by reconstituting cap $5 \mathrm{~A}$ on the plasmid pCapAre, exactly as described in [34]. The fragment was amplified employing genomic DNA of S. aureus SA137/93G 


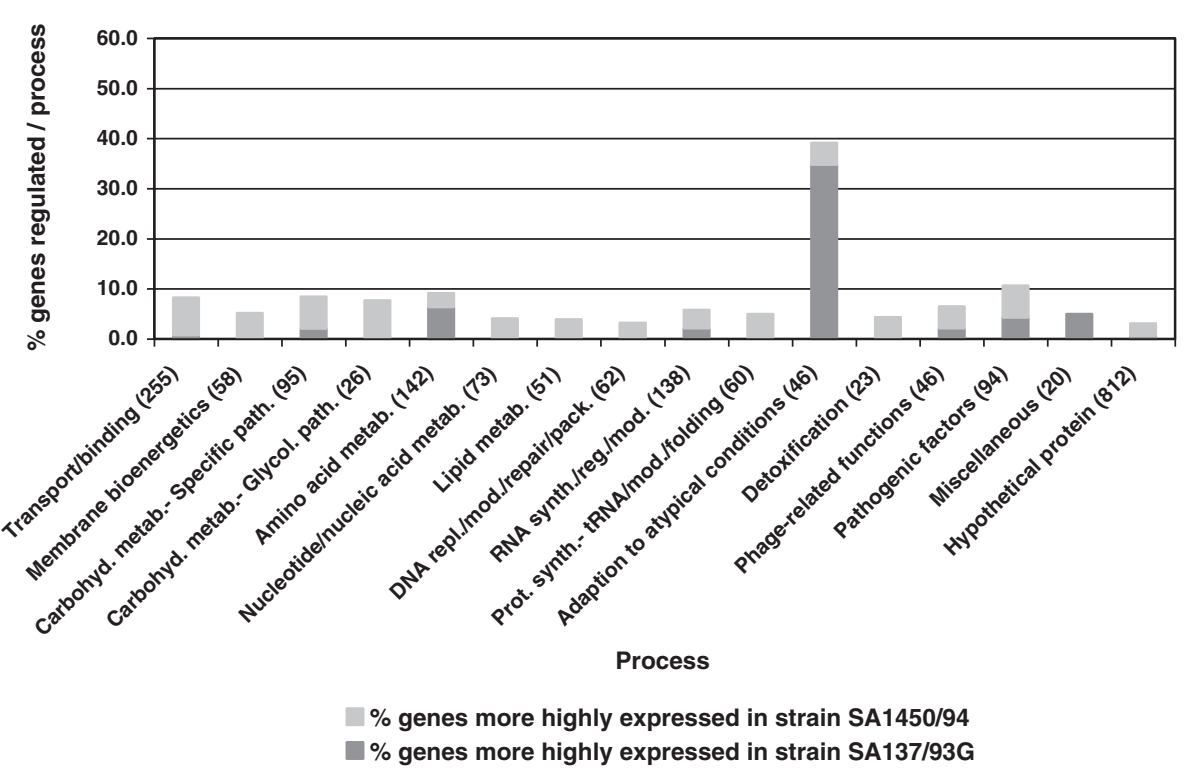

Figure 1 Transcription profiling: comparison of transcriptomes $\left(\mathrm{OD}_{600}=0.8-1.0\right)$ of VISA strain SA137/93G and the related VSSA strain SA1450/94. The regulated genes are represented as percentage of all genes constituting a process category. The number of genes per process category is shown in brackets.

as a template and the primers pCapAreconfor (GCA GAGCTCGCATTTGAA) and pCapAreconrev (CCAAT GATTAAGCTTGATAGTCC). These primers harbour the natural cleavage sites that had been used for complementation previously [34].

\section{Results and discussion}

\section{Comparative transcriptomics}

The clinical VISA isolate SA137/93A and the type strain of the Iberian clone in Germany ('Northern German epidemic strain') SA1450/94 showed identical PFGE patterns [43] and MLST types (ST247). In order to further confirm that SA1450/94 is a suitable control strain, chromosomal DNA of SA137/93A was competitively hybridised to that of SA1450/94 and SA137/93G, respectively. The microarray results showed that all ORFs present in the VISA strain SA137/93A were also present in strain SA1450/94. In addition, the competitive hybridisation precisely reflected the deletion in the mutant SA137/93G [4].
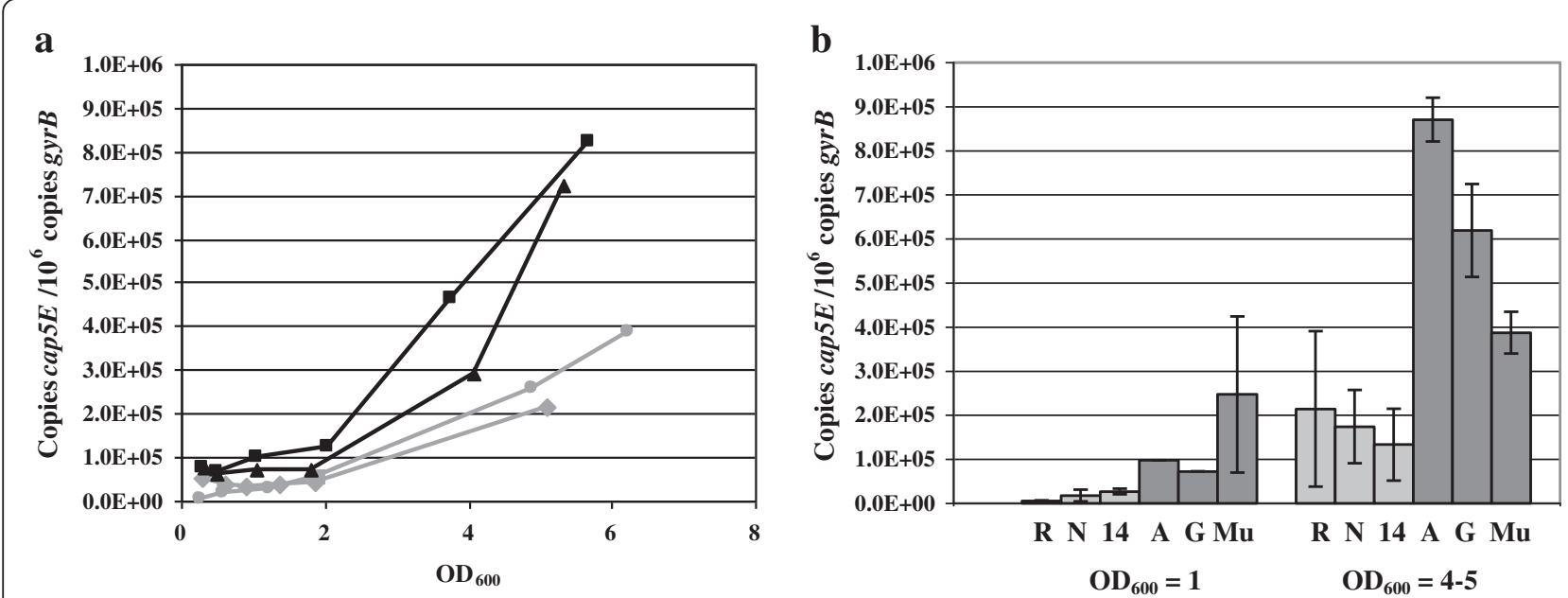

Figure 2 Transcript quantification of the essential capsule biosynthesis gene cap5E by real time PCR. a) Transcript amounts of cap5E throughout the growth curve of hVISA SA137/93A (filled square), VISA SA137/93G (filled triangle), VSSA Newman (filled circle) and VSSA SA1450/ 94 (filled diamond) indicated as copy number per $10^{6}$ copies of the housekeeping gene gyrB. $\mathbf{b}$ ) Transcript amounts of cap5E of VSSA strains (R: Reynolds*, N: Newman, 14: SA1450/94) and VISA strains (A: SA137/93A*, G: SA137/93G*, Mu: Mu50) at $\mathrm{OD}_{600}=1$ and $\mathrm{OD}_{600}=4-5$ indicated as copy number per $10^{6}$ copies of gyrB. ${ }^{*}$ Error bars are not visible at $\mathrm{OD}_{600}=1$ because of minimal data variations. 
Comparative transcriptomics of the hVISA isolate SA137/93A and SA1450/94 revealed that there were only 15 genes showing a higher expression level in the hVISA strain (2- to12-fold; see Additional file 1: gene expression data.pdf, Additional file 1: Table S1). The yycFGHI-operon [27] and three genes of the type 5/8 capsule biosynthesis gene cluster $(c a p B, c a p C, c a p E)$, which showed a 4- to 5fold higher expression, were among the ten genes with a known function in Additional file 1: Table S1. The relatively low number of regulated genes may be due to the fact that the strain shows a heterogeneous phenotype, i.e., only a subpopulation of the culture displays high resistance to vancomycin. Similar results were obtained with the $\mathrm{JH}$ series of mutants; here $\mathrm{JH} 1$ to $\mathrm{JH} 5$ did not show any alterations in gene expression, although resistance had increased [44,45], therefore, this observation was not surprising.

As expected, the transcription profile of the VISA strain SA137/93G differed more strikingly from that of SA1450/94. A total of 124 genes showed at least a twofold change in strain SA137/93G (2- to 13.7-fold; see Additional file 1: gene expression data.pdf, Additional file 1: Table S2) compared to SA1450/94. 30.6\% of these genes encoded hypothetical proteins. Figure 1 shows the percentage of regulated genes in the different functional gene classes. Only one category of genes, "adaptation to atypical conditions", which comprises genes encoding capsule biosynthesis enzymes, chaperones, heat and cold shock proteins and the $c l p$ protease, was overrepresented among the genes showing higher transcription levels. The 16 genes of the capsular biosynthesis gene cluster capABCDEFGHIJKLMNOP were - on average - six fold up-regulated. Additionally, a more than twofold increase in transcript amounts was found for the gene encoding AlsS, which is involved in formation of acetoine from pyruvate and influences the regulation of autolysis [46], the urease operon and two ORFs of the ica gene cluster. All of the above mentioned genes have also been found to be up-regulated under mildly acidic conditions [47]. Since some of the genes with lower expression levels in strain SA137/93G were also down-regulated under acidic conditions, part of the expression profile might be the consequence of differences in the $\mathrm{pH}$ decrease of the glucose-containing BHI medium that was used for cultivating the strains. This hypothesis is supported by the finding of Nelson et al. [48] indicating that an impaired catabolism of acetate seems to be typical for some VISA strains and might result in the up-regulation of urease, which supplies ammonium ions that neutralize the decrease in $\mathrm{pH}$ caused by the formation of acids [49]. In addition, the capsule gene cluster, alsS and SA2262, SA2367 as well as SA2403 are members of the sigB regulon and might indicate an increased SigB activity which has been shown to contribute towards glycopeptide resistance [50]. A more than twofold decrease in expression was observed for 80 genes (2- to 13.7-fold) in the VISA strain SA137/93G in comparison with the susceptible control. In summary, an increased transcription of genes involved in capsule biosynthesis was the only expression pattern that was common to both VISA strains in comparison to the VSSA strain.

\section{Cap5E transcript quantification by real time PCR}

The cap5 and the cap 8 loci are allelic, each comprising 16 genes $(\operatorname{cap} A-P)$ that are transcribed in one orientation with 12 of the 16 genes being nearly identical. The four genes in the central region of the cluster are type-specific and show little homology [51]. The presence of the type 5 gene cluster in the VISA strains and SA1450/94 had been indicated by the microarray results and was confirmed by PCR. In S. aureus, capsule production occurs primarily in the late $\log$ and post-exponential growth phase. It had previously been shown that $S$. aureus CPs are not detectable before the late log growth phase, $2 \mathrm{~h}$ after the transcript increase in the mid log phase [52,53]. For exact quantitative analysis of expression of the CP biosynthetic enzymes and to obtain further insights into capsule production in different growth phases, the transcription level of the essential capsule gene cap5E [34] was determined by real time PCR. Figure 2a shows the expression rate of cap $5 E$ throughout the growth curve of the VISA strains and the controls. The expression
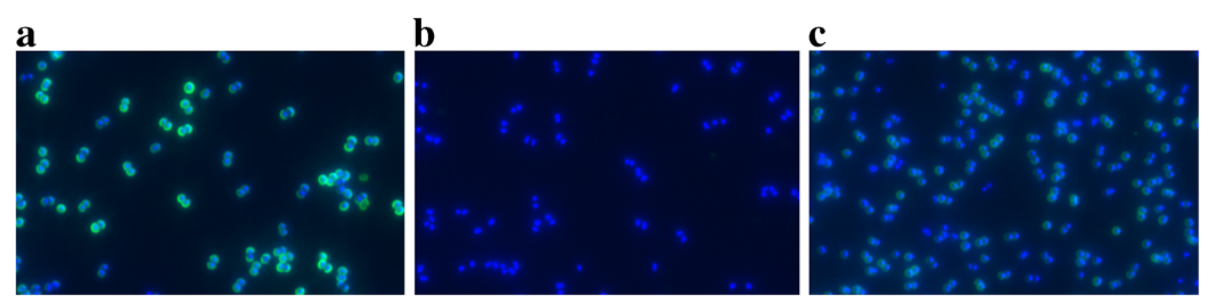

Figure 3 Comparison of CP5 production in a VISA and two VSSA strains. CP5 was labelled by immunofluorescence (CY3, green). As a control, all cells were stained using DAPI (blue). Cells were grown for $6 \mathrm{~h}$ in LB at $37^{\circ} \mathrm{C}$. a) VISA SA137/93G; b) control strain SA 1450/94; c) S. aureus Newman. 
patterns during growth were similar in all tested strains. A strong increase of capsule expression occurred in the post-exponential growth phase after the culture reached an optical density of 2 (Figure 2a) in VSSA and VISA strains, and the basal expression level in strain SA137/93A and SA137/93G was already elevated during the early growth phase. Furthermore, an increase of cap $5 E$ gene transcription could be observed in the stationary growth phase in the VISA strains, with a 2- to 3-fold increased expression level at an $\mathrm{OD}_{600}$ of about 5. A lower transcription in VSSA strains in exponential and stationary phase could be confirmed when strain Reynolds and Newman were included into the measurements (Figure 2b). These findings were consistent with the primary microarraybased data of this study and expression data of $S$. aureus Mu50 [10].

The CPs of Klebsiella pneumoniae were found to contribute to resistance to cationic defensins, lactoferrin, protamine sulfate and polymyxin $\mathrm{B}$, and in this context, the capsule was assumed to protect bacteria by limiting the interaction of the antimicrobial peptides with the surface [54]. Later, similar results were obtained with polysaccharides from Streptococcus pneumoniae and alginate from Pseudomonas aeruginosa [55]. A possible role of CPs in vancomycin resistance has repeatedly been discussed in the literature. Boyle-Vavra et al. found that susceptible passage revertants of the CP5 producing VISA isolates MI, $\mathrm{NJ}$ and $\mathrm{PC}$ were no longer $\mathrm{CP}$ typable, while passaging in presence of vancomycin retained the CP phenotype [56]. Besides, comparative expression profiling experiments on VISA isolates Mu50, MI, JH9 and their respective susceptible parent or mutant strains showed that some (but not necessarily all) of the genes of the type 5 capsule were more highly expressed in the VISA strains [10,45]. Enhanced capsule production in other VISA was also reported [57] and deletion of the yabJ-spoVG operon affected glycopeptide susceptibility and capsule production in $S$. aureus simultaneously [50]. Taken together, these findings encouraged us to further investigate the role of CPs in vancomycin resistance.

\section{Detection of the capsule by immunofluorescence}

Production of CP5 was analysed by immunofluorescent labelling of cells of SA137/93G and the susceptible strains SA1450/94 and Newman after $6 \mathrm{~h}$ of incubation in LB. The results revealed that the VISA strain produced higher amounts of CP5 than SA1450/94 and S. aureus Newman (Figure 3).

\section{Sequence of the capsule promoter of VISA strains and SA $1450 / 94$}

In order to detect possible changes in the promoter of the capsule gene cluster that might be responsible for an elevated expression in the VISA strains, a region covering 400 bp upstream of cap5A was sequenced. The complete sequences were identical to that published for $S$. aureus COL (ST250), which is a close relative of the Iberian strain, and S. aureus RF122. The promoter sequence of the cap5 gene cluster and the inverted repeats that constitute the operator $[58,59]$ were identical to that of the first seven published genomes. Unexpectedly, the control strain SA1450/94 showed an insertion of IS256 into the first gene of the capsule gene cluster cap5A1. The IS element was located 50 bp downstream of the ATG start codon and oriented in an antisense direction. Cap5A1 encodes a membrane protein that is part of the protein kinase Cap5A1/Cap5B2, which is needed for phosphorylation of Cap5O [60]. In spite of this, in in vitro experiments Cap5A1 is not essential for activation of Cap5O since a paralogue of Cap5A1, Cap5A2 is encoded by SA2457 and able to activate the kinase subunit Cap5B2 [60]; this is also demonstrated by the fact that SA1450/94 was able to produce capsule, albeit at low levels, in overnight cultures (data not shown).

\section{The effect of capsule on vancomycin resistance in VISA} Initial attempts to knock out capsule production in the VISA strains resulted in mutants that could not be complemented because they harboured background mutations in regulatory genes that are necessary for capsule production and influence glycopeptide susceptibility
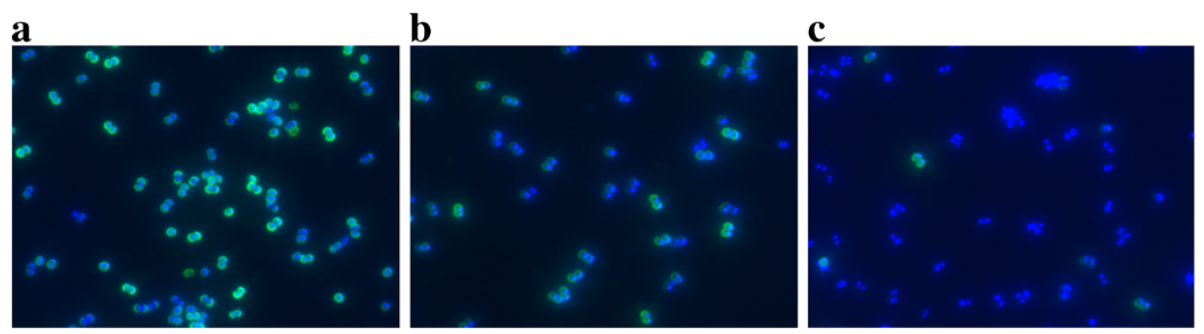

Figure 4 Suppression of capsule formation by expression of cap5D-antisense RNA. CP5 was labelled by immunofluorescence (CY3, green), the cells were stained using DAPI (blue). Cells were grown for $6 \mathrm{~h}$ in LB at $37^{\circ} \mathrm{C}$. a) S. aureus SA137/93G (control); b) S. aureus SA137/93G harbouring pCapDvorne in the absence of xylose and c) grown in the presence of $50 \mathrm{mM}$ xylose. 


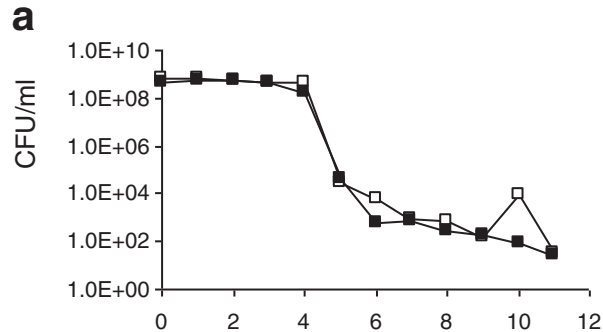

b

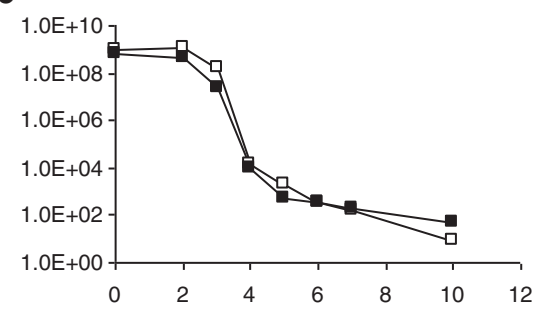

C

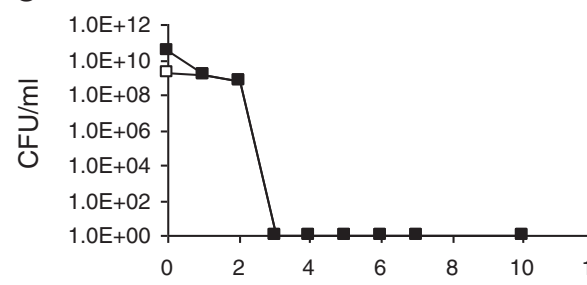

d

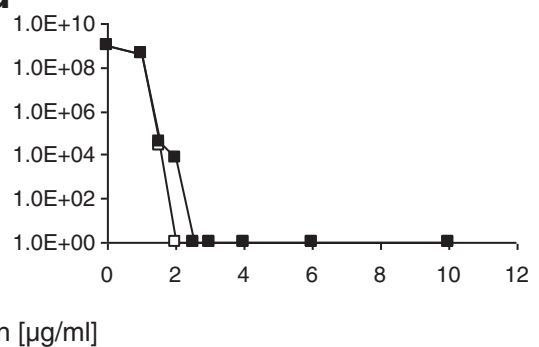

Figure 5 Population analyses of different strains in the presence or absence of capsule. a) S. aureus SA137/93G harbouring pCapDvorne grown on BHI agar in the absence of xylose (capsule; $\square$ ) or in the presence of xylose (no capsule; a ); b) S. aureus SA137/93G harbouring pCapDvorne grown on TSA without glucose in the absence ( $\square$ ) or in the presence of xylose ( $\mathbf{a}$ ); c) S. aureus HG001 ( $\square$ ) and S. aureus HG001 harbouring pcap5E ( $\mathbf{n}$ ) which leads to reconstitution of capsule biosynthesis on BHI agar; $\mathbf{d}$ ) S. aureus Newman harbouring an insertion of pMUTIN4 in the capsule promoter grown on MH agar in the absence ( $\square$ ) and the presence ( $\mathbf{a})$ of 1 mM IPTG.

(rsbU, agr), e.g., inactivation of rsbU led to an increase in vancomycin susceptibility in our isolates even if capsule biosynthesis had been reconstituted. Therefore, we chose an antisense approach. An N-terminal $166 \mathrm{bp}$ fragment of cap5D was ligated to pEPSA5 in antisense direction and transformed into S. aureus 137/93G. We chose another region than that described in [30] since antisense RNA expression from this fragment had exerted growth-inhibitory effects. Capsule formation was analyzed by immunofluorescence in the absence and presence of $50 \mathrm{mM}$ xylose in different media (LB, BHI and CYPG [61]) after $6 \mathrm{~h}$ of incubation. Figure 4 shows that after only $6 \mathrm{~h}$ of incubation, capsule formation in the wildtype SA137/93G is relatively strong even in LB (Figure 4c), and that the capsule formation is somewhat decreased in the presence of the plasmid even in the absence of xylose (Figure 4b). Addition of $50 \mathrm{mM}$ xylose (but not $12.5 \mathrm{mM}$ ) led to a full repression of capsule biosynthesis (Figure 4c) in all tested media with the exception of a few cells that had obviously been able to eliminate the plasmid.

In MIC determinations in LB/CM 34, no significant difference in vancomycin resistance was observed after expression of antisense RNA in S. aureus SA137/93G. The value of $1.5 \pm 0.4 \mathrm{mg} / \mathrm{L}$ vancomycin obtained for encapsulated strains grown in the absence of xylose was lowered to $1.3 \pm 0.3 \mathrm{mg} / \mathrm{L}$ vancomycin for capsule-free cells incubated in the presence of xylose.

Intermediate vancomycin susceptibility of VISA strains is most easily demonstrated in population analyses on BHI, which is the medium that yields the highest vancomycin
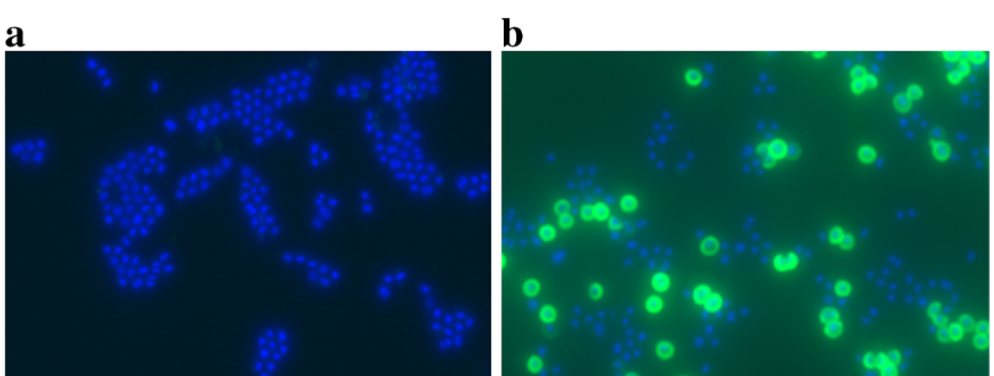

Figure 6 Repair of capsule formation in S. aureus HG001. CP5 was labelled by immunofluorescence (CY3, green), the cells were stained using DAPI (blue). Cells were grown in TSB medium overnight at $37^{\circ} \mathrm{C}$. a) S. aureus HG001 (control); b) S. aureus HG001 pCap5E, in which capsule production has been reconstituted. 

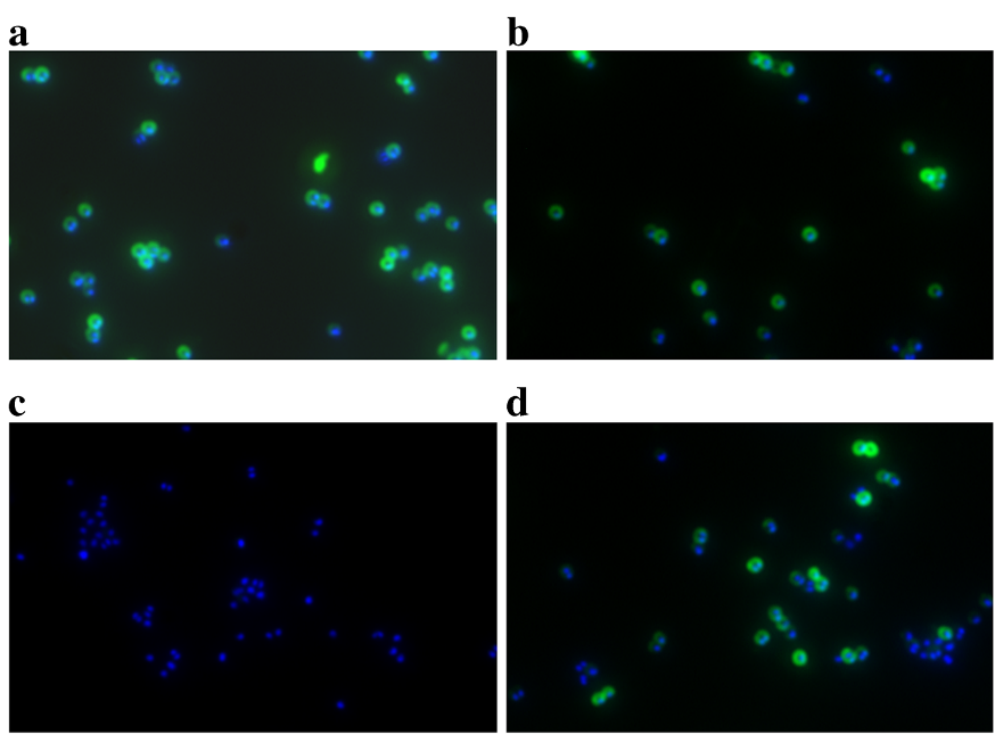

d

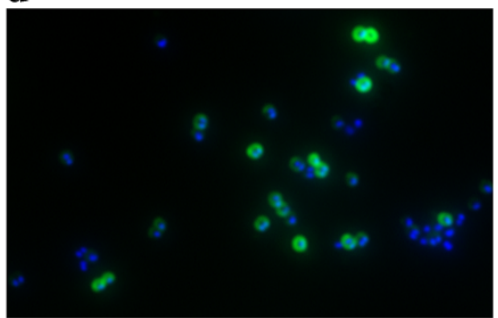

Figure 7 Induction of capsule production by IPTG in S. aureus Newman-132. CP5 was labelled by immunofluorescence (CY3, green), the cells were stained using DAPI (blue). Cells were grown for $6 \mathrm{~h}$ in MH medium at $37^{\circ} \mathrm{C}$. a) S. aureus Newman (control) b) S. aureus Newman in the presence of $0.5 \mathrm{mM}$ IPTG; c) S. aureus Newman-132 harbouring pMUTIN4 in the capsule promoter in the absence of IPTG and d) S. aureus Newman-132 harbouring PMUTIN4 in the capsule promoter after induction with IPTG.

MICs and therefore should be the most sensitive medium. Again there was no difference in the population analyses of clones grown in the absence or presence of xylose (Figure 5a). Experiments in TSA-G (TSA without glucose) yielded similar results (Figure $5 \mathrm{~b})$.

\section{The effect of the capsule on vancomycin resistance in VSSA}

In addition to the VISA strain, the effect of the capsule on vancomycin resistance in three vancomycin susceptible strains producing CP5 was investigated. All strains of the RN1 (NCTC 8325) lineage harbour a mutation in cap5E that leads to inactivation of capsule biosynthesis. Furthermore a deletion in rsbu leads to a very low activity of sigma $B$ which however is needed for the efficient transcription of the capsule biosynthetic genes [50]. As described in [34], capsule production was reconstituted into S. aureus HG001 (rsbU repaired) by introduction of a plasmid carrying a
cap5E gene amplified from S. aureus Newman (Figure 6). Again the population showed a heterogeneous phenotype in immunofluorescence experiments. However, in population analyses no increase in resistance against vancomycin could be detected (Figure $5 \mathrm{c}$ ).

An S. aureus Newman clone with the capsule promoter under control of Pspac was capsule negative in the absence of inducer, but heterogeneous capsule production could be achieved by addition of IPTG to media that did not contain glucose, e.g., $\mathrm{MH}$ (Figure 7). In population analyses with these cells, no significant difference in vancomycin resistance on $\mathrm{MH}$ agar was visible (Figure $5 \mathrm{~d}$ ).

As capsule production in SA1450/94 might be impaired by the insertion of IS256 described above, it was attempted to reconstitute CP5 production. In $S$. aureus Newman insertion of Tn916 into cap5A1 could be repaired by complementation of cap5A1 in trans [34]. Therefore, a similar construct (pCapAre) was introduced into SA1450/94,
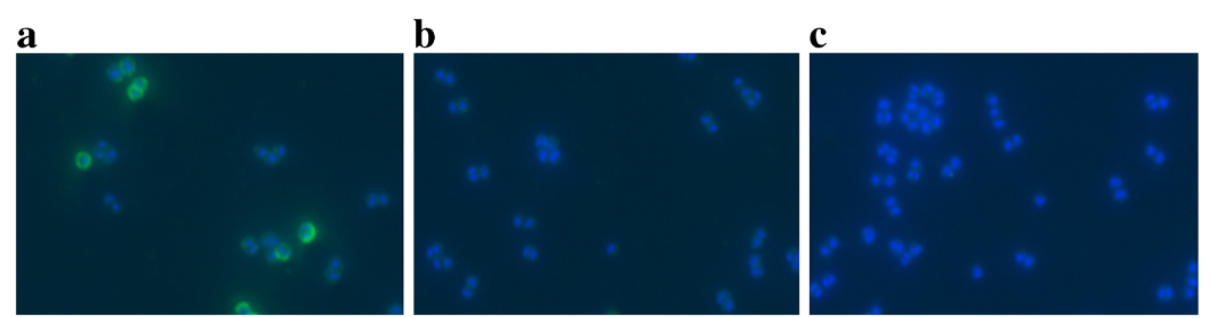

Figure 8 Capsule production of different S. aureus SA1450/94 clones. CP5 was labelled by immunofluorescence (CY3, green), the cells were stained using DAPI (blue). Cells were grown for 6 hours in BHI medium at $37^{\circ} \mathrm{C}$. a) S. aureus SA1450/94 harbouring pCapAre, which has reconstituted capsule production; b) SA1450/94 (control) and c) SA1450/94 harbouring pCU1 (vector control). 
which increased capsule production compared to the parent strain (Figure 8). However, full capsule production was not achieved and the vancomycin MIC of the clone remained unchanged compared to SA1450/94.

Furthermore, a capsule knockout mutant of strain Reynolds had previously been tested against vancomycin, and no differences in susceptibility to vancomycin were recorded [62]. Population analyses in our laboratory confirmed this result (data not shown).

\section{Effect of capsule material on the susceptibility of staphylococci to vancomycin}

In order to test whether capsule material is able to interact with or bind to vancomycin, crude capsular material was prepared from S. aureus 137/93G and S. aureus NCTC 8325 (negative control; as shown in Figure 6 for S. aureus HG001, the strains of this lineage do not produce a capsule unless cap $5 E$ is repaired). Cell wall teichoic acid that might contaminate the extracts was removed by periodate oxidation. The material was added to MIC determinations using S. aureus NCTC8325 and S. aureus SG511 as indicator strains in $\mathrm{MH}$ medium. There was no significant difference in the MIC values between the extract containing capsular material and the controls for S. aureus SG511, however a small effect $(0.7 \mathrm{mg} / \mathrm{L}$ increase in the MIC) was visible with $S$. aureus NCTC8325 and the extract of $S$. aureus SA137/93G. The test was repeated 8 times with two different preparations of the capsular material; an additional DNase and RNase digest did not influence the result. While we cannot explain this difference, the fact that no increase in the MIC was visible with the more susceptible indicator strain strongly indicated that the type 5 capsular material did not neutralise the effect of vancomycin in this assay. The experiment was repeated with purified CP5 $(32 \mu \mathrm{g} / \mathrm{ml})$ in MIC determinations in $\mathrm{MH}$ medium. Both indicator strains did not show any alterations in susceptibility to vancomycin, which confirmed the above result.

\section{Conclusions}

Although an increased transcription of the capsular gene cluster has been observed for several VISA strains, the type 5 capsule does not seem to play a significant role in the resistance mechanism of $S$. aureus 137/93G. It may therefore be assumed that - at least in the strain investigated here an increased or uniform transcription of the capsule gene cluster is a phenomenon that accompanies vancomycin resistance, perhaps a by-product of a relatively high SigB activity in S. aureus 137/93G, indicated by the intense yellow colour of this strain, that might contribute to glycopeptide resistance [50] or an overflow from an activated cell wall metabolism [1], rather than being the cause for vancomycin resistance.

\section{Additional file}

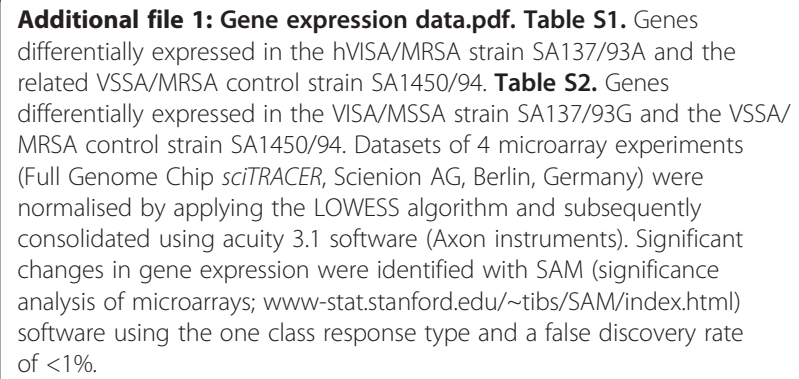

Competing interests

The authors declare that they have no competing interests.

\section{Authors' contributions}

AJ designed the study, carried out the microarray and GRT-PCR experiments, performed susceptibility experiments and drafted the manuscript. CS constructed mutants in S. aureus SA137/93G, SA1450/94 and S. aureus HG001 and performed susceptibility experiments. WS, CW and CG carried out the immunofluorescence visualisation of the capsule polysaccharides, integrated the plasmid PMUTIN4 into the capsule promoter of S. aureus Newman and contributed to GRT-PCR experiments. JL gave critical advice for the design of the study, provided capsular antibody, purified CP5, and the Reynolds $\mathrm{CP}+/ \mathrm{CP}$ - strain pair. MT participated in mutant construction. GB conceived the study, participated in its design and drafted the manuscript. All authors read and approved the final manuscript.

\section{Acknowledgements}

This work was supported by the Bundesministerium für Wissenschaft und Forschung (PTJ-BIO/03U213B and PTJ-BIO/0313801 F) and the DFG (Bi504/8-1,2) to $G B$ and the SFB766, project A7 to CW. V. Fuchs is thanked for expert technical assistance. We thank T. Roemer for supplying pEPSA5.

\section{Author details}

${ }^{1}$ Institut für Medizinische Mikrobiologie, Immunologie und Parasitologie, Universitätsklinikum Bonn, Sigmund-Freud-Str. 25, D-53105, Bonn, Germany. ${ }^{2}$ Interfakultäres Institut für Mikrobiologie und Infektionsmedizin, Universität Tübingen, D-72076, Tübingen, Germany. ${ }^{3}$ Channing Laboratory, Brigham and Women's Hospital, Harvard Medical School, Boston 02115, USA

Received: 18 January 2013 Accepted: 4 March 2013

Published: 22 March 2013

\section{References}

1. Hanaki H, Kuwahara-Arai K, Boyle-Vavra S, Daum RS, Labischinski H, Hiramatsu K: Activated cell-wall synthesis is associated with vancomycin resistance in methicillin-resistant Staphylococcus aureus clinical strains Mu3 and Mu50. J Antimicrob Chemother 1998, 42:199-209.

2. Cui L, Iwamoto A, Lian JQ, Neoh HM, Maruyama T, Horikawa Y, et al: Novel mechanism of antibiotic resistance originating in vancomycinintermediate Staphylococcus aureus. Antimicrob Agents Chemother 2006 50:428-438

3. Cui L, Ma X, Sato K, Okuma K, Tenover FC, Mamizuka EM, et al: Cell wall thickening is a common feature of vancomycin resistance in Staphylococcus aureus. J Clin Microbiol 2003, 41:5-14.

4. Reipert A, Ehlert K, Kast T, Bierbaum G: Morphological and genetic differences in two isogenic Staphylococcus aureus strains with decreased susceptibilities to vancomycin. Antimicrob Agents Chemother 2003, 47:568-576

5. Sieradzki K, Tomasz A: Inhibition of cell wall turnover and autolysis by vancomycin in a highly vancomycin-resistant mutant of Staphylococcus aureus. J Bacteriol 1997, 179:2557-2566.

6. Sieradzki K, Tomasz A: Inhibition of the autolytic system by vancomycin causes mimicry of vancomycin-intermediate Staphylococcus aureus-type resistance, cell concentration dependence of the MIC, and antibiotic 
tolerance in vancomycin-susceptible S. aureus. Antimicrob Agents Chemother 2006, 50:527-533.

7. Utaida S, Pfeltz RF, Jayaswal RK, Wilkinson BJ: Autolytic properties of glycopeptide-intermediate Staphylococcus aureus Mu50. Antimicrob Agents Chemother 2006, 50:1541-1545.

8. Cui L, Murakami H, Kuwahara-Arai K, Hanaki H, Hiramatsu K: Contribution of a thickened cell wall and its glutamine nonamidated component to the vancomycin resistance expressed by Staphylococcus aureus Mu50. Antimicrob Agents Chemother 2000, 44:2276-2285.

9. Howden BP, Davies JK, Johnson PD, Stinear TP, Grayson ML: Reduced vancomycin susceptibility in Staphylococcus aureus, including vancomycin-intermediate and heterogeneous vancomycin-intermediate strains: resistance mechanisms, laboratory detection, and clinical implications. Clin Microbiol Rev 2010, 23:99-139.

10. Cui L, Lian JQ, Neoh HM, Reyes E, Hiramatsu K: DNA microarray-based identification of genes associated with glycopeptide resistance in Staphylococcus aureus. Antimicrob Agents Chemother 2005, 49:3404-3413.

11. Kuroda M, Kuwahara-Arai K, Hiramatsu K: Identification of the up- and down-regulated genes in vancomycin-resistant Staphylococcus aureus strains Mu3 and Mu50 by cDNA differential hybridization method. Biochem Biophys Res Commun 2000, 269:485-490.

12. Mongodin E, Finan J, Climo MW, Rosato A, Gill S, Archer GL: Microarray transcription analysis of clinical Staphylococcus aureus isolates resistant to vancomycin. J Bacterio/ 2003, 185:4638-4643.

13. Cui L, Neoh HM, Shoji M, Hiramatsu K: Contribution of vraSR and graSR point mutations to vancomycin resistance in vancomycin-intermediate Staphylococcus aureus. Antimicrob Agents Chemother 2009, 53:1231-1234.

14. Howden BP, McEvoy CR, Allen DL, Chua K, Gao W, Harrison PF, et al: Evolution of multidrug resistance during Staphylococcus aureus infection involves mutation of the essential two component regulator WaIKR. PLOS Pathog 2011, 7:e1002359.

15. Doddangoudar VC, Boost MV, Tsang DN, O'Donoghue MM: Tracking changes in the vraSR and graSR two component regulatory systems during the development and loss of vancomycin non-susceptibility in a clinical isolate. Clin Microbiol Infect 2011, 17:1268-1272.

16. Gardete S, Kim C, Hartmann BM, Mwangi M, Roux CM, Dunman PM, et al: Genetic pathway in acquisition and loss of vancomycin resistance in a methicillin resistant Staphylococcus aureus (MRSA) strain of clonal type USA300. PLoS Pathog 2012, 8:e1002505.

17. Hafer C, Lin Y, Kornblum J, Lowy FD, Uhlemann AC: Contribution of selected gene mutations to resistance in clinical isolates of vancomycinintermediate Staphylococcus aureus. Antimicrob Agents Chemother 2012, 56:5845-5851.

18. Neoh HM, Cui L, Yuzawa H, Takeuchi F, Matsuo M, Hiramatsu K: Mutated response regulator graR is responsible for phenotypic conversion of Staphylococcus aureus from heterogeneous vancomycin-intermediate resistance to vancomycin-intermediate resistance. Antimicrob Agents Chemother 2008, 52:45-53.

19. Meehl M, Herbert S, Götz F, Cheung A: Interaction of the GraRS twocomponent system with the VraFG $A B C$ transporter to support vancomycin-intermediate resistance in Staphylococcus aureus. Antimicrob Agents Chemother 2007, 51:2679-2689.

20. Cui L, Isii T, Fukuda M, Ochiai T, Neoh HM, Camargo IL, et al: An RpoB mutation confers dual heteroresistance to daptomycin and vancomycin in Staphylococcus aureus. Antimicrob Agents Chemother 2010, 54:5222-5233.

21. Watanabe Y, Cui L, Katayama Y, Kozue K, Hiramatsu K: Impact of rpoB mutations on reduced vancomycin susceptibility in Staphylococcus aureus. J Clin Microbiol 2011, 49:2680-2684.

22. Matsuo M, Hishinuma T, Katayama Y, Cui L, Kapi M, Hiramatsu K: Mutation of RNA polymerase beta subunit $(r p o B)$ promotes hVISA-to-VISA phenotypic conversion of strain Mu3. Antimicrob Agents Chemother 2011, 55:4188-4195

23. Passalacqua KD, Satola SW, Crispell EK, Read TD: A mutation in the PP2C phosphatase gene in a Staphylococcus aureus USA300 clinical isolate with reduced susceptibility to vancomycin and daptomycin. Antimicrob Agents Chemother 2012, 56:5212-5223.

24. Jousselin A, Renzoni A, Andrey DO, Monod A, Lew DP, Kelley WL: The posttranslocational chaperone lipoprotein PrsA is involved in both glycopeptide and oxacillin resistance in Staphylococcus aureus. Antimicrob Agents Chemother 2012, 56:3629-3640.
25. Shoji M, Cui L, lizuka R, Komoto A, Neoh HM, Watanabe $Y$, et al: walK and clpP mutations confer reduced vancomycin susceptibility in Staphylococcus aureus. Antimicrob Agents Chemother 2011, 55:3870-3881.

26. Maki H, McCallum N, Bischoff M, Wada A, Berger-Bächi B: tcaA inactivation increases glycopeptide resistance in Staphylococcus aureus. Antimicrob Agents Chemother 2004, 48:1953-1959.

27. Jansen A, Türck M, Szekat C, Nagel M, Clever I, Bierbaum G: Role of insertion elements and yycFG in the development of decreased susceptibility to vancomycin in Staphylococcus aureus. Int J Med Microbiol 2007, 297:205-215.

28. Wada A, Katayama Y, Hiramatsu K, Yokota T: Southern hybridization analysis of the mecA deletion from methicillin-resistant Staphylococcus aureus. Biochem Biophys Res Commun 1991, 176:1319-1325.

29. Hiramatsu K, Hanaki H, Ino T, Yabuta K, Oguri T, Tenover FC: Methicillinresistant Staphylococcus aureus clinical strain with reduced vancomycin susceptibility. J Antimicrob Chemother 1997, 40:135-136.

30. Forsyth RA, Haselbeck RJ, Ohlsen KL, Yamamoto RT, Xu H, Trawick JD, et al: A genome-wide strategy for the identification of essential genes in Staphylococcus aureus. Mol Microbiol 2002, 43:1387-1400.

31. Herbert S, Ziebandt AK, Ohlsen K, Schäfer T, Hecker M, Albrecht D, et al: Repair of global regulators in Staphylococcus aureus 8325 and comparative analysis with other clinical isolates. Infect Immun 2010, 78:2877-2889.

32. Sass $P$, Bierbaum G: Native graS mutation supports the susceptibility of Staphylococcus aureus strain SG511 to antimicrobial peptides. Int J Med Microbiol 2009, 299:313-322.

33. Kreiswirth BN, Löfdahl S, Betley MJ, O'Reilly M, Schlievert PM, Bergdoll MS, et al: The toxic shock syndrome exotoxin structural gene is not detectably transmitted by a prophage. Nature 1983, 305:709-712

34. Wann ER, Dassy B, Fournier JM, Foster TJ: Genetic analysis of the cap5 locus of Staphylococcus aureus. FEMS Microbiol Lett 1999, 170:97-103.

35. Pöhlmann-Dietze $P$, Ulrich M, Kiser KB, Döring G, Lee JC, Fournier JM, et al: Adherence of Staphylococcus aureus to endothelial cells: influence of capsular polysaccharide, global regulator agr, and bacterial growth phase. Infect Immun 2000, 68:4865-4871.

36. Schenk S, Laddaga RA: Improved method for electroporation of Staphylococcus aureus. FEMS Microb Lett 1992, 94:133-138.

37. Berger-Bächi B, Kohler ML: A novel site on the chromosome of Staphylococcus aureus influencing the level of methicillin resistance: genetic mapping. FEMS Microbiol Lett 1983, 20:305-309.

38. Vagner V, Dervyn E, Ehrlich SD: A vector for systematic gene inactivation in Bacillus subtilis. Microbiology 1998, 144:3097-3104.

39. Lee JC, Michon F, Perez NE, Hopkins CA, Pier GB: Chemical characterization and immunogenicity of capsular polysaccharide isolated from mucoid Staphylococcus aureus. Infect Immun 1987, 55:2191-2197.

40. Cook J, Hepler R, Pancari G, Kuklin N, Fan H, Wang XM, et al: Staphylococcus aureus capsule type 8 antibodies provide inconsistent efficacy in murine models of staphylococcal infection. Hum Vaccin 2009, 5:254-263.

41. Tzianabos AO, Wang JY, Lee JC: Structural rationale for the modulation of abscess formation by Staphylococcus aureus capsular polysaccharides. Proc Natl Acad Sci USA 2001, 98:9365-9370.

42. Goerke C, Esser S, Kümmel M, Wolz C: Staphylococcus aureus strain designation by agr and cap polymorphism typing and delineation of agr diversification by sequence analysis. Int J Med Microbiol 2005, 295:67-75.

43. Bierbaum G, Fuchs K, Lenz W, Szekat C, Sahl HG: Presence of Staphylococcus aureus with reduced susceptibility to vancomycin in Germany. Eur J Clin Microbiol Infect Dis 1999, 18:691-696.

44. Sieradzki K, Leski T, Dick J, Borio L, Tomasz A: Evolution of a vancomycinintermediate Staphylococcus aureus strain in vivo: multiple changes in the antibiotic resistance phenotypes of a single lineage of methicillinresistant S. aureus under the impact of antibiotics administered for chemotherapy. J Clin Microbiol 2003, 41:1687-1693.

45. McAleese F, Wu SW, Sieradzki K, Dunman P, Murphy E, Projan S, et al: Overexpression of genes of the cell wall stimulon in clinical isolates of Staphylococcus aureus exhibiting vancomycin-intermediate-S. aureustype resistance to vancomycin. J Bacterio/ 2006, 188:1120-1133.

46. Yang SJ, Dunman PM, Projan SJ, Bayles KW: Characterization of the Staphylococcus aureus CidR regulon: elucidation of a novel role for acetoin metabolism in cell death and lysis. Mol Microbiol 2006, 60:458-468. 
47. Weinrick B, Dunman PM, McAleese F, Murphy E, Projan SJ, Fang Y, et al: Effect of mild acid on gene expression in Staphylococcus aureus. J Bacteriol 2004, 186:8407-8423.

48. Nelson JL, Rice KC, Slater SR, Fox PM, Archer GL, Bayles KW, et al: Vancomycin-intermediate Staphylococcus aureus strains have impaired acetate catabolism: implications for polysaccharide intercellular adhesin synthesis and autolysis. Antimicrob Agents Chemother 2007, 51:616-622.

49. Booth IR: Regulation of cytoplasmic pH in bacteria. Microbiol Rev 1985, 49:359-378.

50. Schulthess B, Meier S, Homerova D, Goerke C, Wolz C, Kormanec J, et al: Functional characterization of the sigmaB-dependent yabJ-spoVG operon in Staphylococcus aureus: role in methicillin and glycopeptide resistance. Antimicrob Agents Chemother 2009, 53:1832-1839.

51. Sau S, Bhasin N, Wann ER, Lee JC, Foster TJ, Lee CY: The Staphylococcus aureus allelic genetic loci for serotype 5 and 8 capsule expression contain the type-specific genes flanked by common genes. Microbiology 1997, 143:2395-2405.

52. Luong T, Sau S, Gomez M, Lee JC, Lee CY: Regulation of Staphylococcus aureus capsular polysaccharide expression by agr and sarA. Infect Immun 2002, 70:444-450

53. O'Riordan K, Lee JC: Staphylococcus aureus capsular polysaccharides. Clin Microbiol Rev 2004, 17:218-234.

54. Campos MA, Vargas MA, Regueiro V, Llompart CM, Alberti S, Bengoechea JA: Capsule polysaccharide mediates bacterial resistance to antimicrobial peptides. Infect Immun 2004, 72:7107-7114.

55. Llobet $\mathrm{E}$, Tomas JM, Bengoechea JA: Capsule polysaccharide is a bacterial decoy for antimicrobial peptides. Microbiology 2008, 154:3877-3886.

56. Boyle-Vavra S, Berke SK, Lee JC, Daum RS: Reversion of the glycopeptide resistance phenotype in Staphylococcus aureus clinical isolates. Antimicrob Agents Chemother 2000, 44:272-277.

57. Howden BP, Smith DJ, Mansell A, Johnson PD, Ward PB, Stinear TP, et al: Different bacterial gene expression patterns and attenuated host immune responses are associated with the evolution of low-level vancomycin resistance during persistent methicillin-resistant Staphylococcus aureus bacteraemia. BMC Microbiol 2008, 8:39.

58. Ouyang S, Sau S, Lee CY: Promoter analysis of the cap8 operon, involved in type 8 capsular polysaccharide production in Staphylococcus aureus. J Bacteriol 1999, 181:2492-2500.

59. Pohl K, Francois P, Stenz L, Schlink F, Geiger T, Herbert S, et al: CodY in Staphylococcus aureus: a regulatory link between metabolism and virulence gene expression. J Bacteriol 2009, 191:2953-2963.

60. Soulat D, Grangeasse C, Vaganay E, Cozzone AJ, Duclos B: UDP-acetylmannosamine dehydrogenase is an endogenous protein substrate of Staphylococcus aureus protein-tyrosine kinase activity. J Mol Microbiol Biotechnol 2007, 13:45-54.

61. Novick RP: Genetic systems in staphylococci. Methods Enzymol 1991, 204:587-636.

62. Seaman P, Day M, Russell AD, Ochs D: Susceptibility of capsular Staphylococcus aureus strains to some antibiotics, triclosan and cationic biocides. J Antimicrob Chemother 2004, 54:696-698.

doi:10.1186/1471-2180-13-65

Cite this article as: Jansen et al:: Production of capsular polysaccharide does not influence Staphylococcus aureus vancomycin susceptibility. BMC Microbiology 2013 13:65.

\section{Submit your next manuscript to BioMed Central and take full advantage of:}

- Convenient online submission

- Thorough peer review

- No space constraints or color figure charges

- Immediate publication on acceptance

- Inclusion in PubMed, CAS, Scopus and Google Scholar

- Research which is freely available for redistribution

Submit your manuscript at www.biomedcentral.com/submit 